\title{
Scaling Relation between Sunyaev-Zel'dovich Effect and X-ray Luminosity and Scale-Free Evolution of Cosmic Baryon Field
}

\author{
Qiang Yuan ${ }^{\mathrm{a}, \mathrm{b}} \mathrm{Hao-Yi}$ Wan ${ }^{\mathrm{a}}$ Tong-Jie Zhang ${ }^{\mathrm{a}, \mathrm{c}, \mathrm{d}, *}$ Ji-Ren Liu ${ }^{\mathrm{d}}$ \\ Long-Long Feng e,f Li-Zhi Fang ${ }^{d}$ \\ ${ }^{a}$ Department of Astronomy, Beijing Normal University, Beijing, 100875, \\ P.R.China \\ ${ }^{\mathrm{b}}$ Key Laboratory of Particle Astrophysics, Institute of High Energy Physics, \\ Chinese Academy of Sciences, Beijing 100049, P.R.China \\ ${ }^{\mathrm{c}}$ Kavli Institute for Theoretical Physics China, Institute of Theoretical Physics, \\ Chinese Academy of Sciences (KITPC/ITP-CAS), P.O.Box 2735, Beijing 100080, \\ P.R. China \\ ${ }^{\mathrm{d}}$ Department of Physics, University of Arizona, Tucson, AZ 85721 \\ e Purple Mountain Observatory, Nanjing 210008, P.R. China \\ ${ }^{\mathrm{f}}$ National Astronomical Observatories, Chinese Academy of Science, Chao-Yang \\ District, Beijing, 100012, P.R. China
}

\begin{abstract}
It has been revealed recently that, in the scale free range, i.e. from the scale of the onset of nonlinear evolution to the scale of dissipation, the velocity and mass density fields of cosmic baryon fluid are extremely well described by the self-similar log-Poisson hierarchy. As a consequence of this evolution, the relations among various physical quantities of cosmic baryon fluid should be scale invariant, if the physical quantities are measured in cells on scales larger than the dissipation scale, regardless the baryon fluid is in virialized dark halo, or in pre-virialized state. We examine this property with the relation between the Compton parameter of the thermal Sunyaev-Zel'dovich effect, $y(r)$, and X-ray luminosity, $L_{\mathrm{x}}(r)$, where $r$ being the scale of regions in which $y$ and $L_{\mathrm{x}}$ are measured. According to the self-similar hierarchical scenario of nonlinear evolution, one should expect that 1.) in the $y(r)-L_{x}(r)$ relation, $y(r)=10^{A(r)}\left[L_{\mathrm{x}}(r)\right]^{\alpha(r)}$, the coefficients $A(r)$ and $\alpha(r)$ are scale-invariant; 2.) The relation $y(r)=10^{A(r)}\left[L_{\mathrm{x}}(r)\right]^{\alpha(r)}$ given by cells containing collapsed objects is also available for cells without collapsed objects, only if $r$ is larger than the dissipation scale. These two predictions are well established with a scale decomposition analysis of observed data, and a comparison of observed $y(r)-L_{x}(r)$ relation with hydrodynamic simulation samples. The implication of this result on the characteristic scales of non-gravitational heating is also addressed.
\end{abstract}


Key words: cosmology: theory, large-scale structure of universe, X-rays: galaxies: clusters, hydrodynamics, methods: numerical

PACS: 95.30.Jx, 07.05.Tp, 98.80.-k

\section{Introduction}

Scaling relation of dimensional quantities is very powerful to reveal the dynamical feature of various physical systems. There has been a considerable effort devoting to study the correlations and scaling laws of various observable quantities of galaxy clusters. Since virialized self-gravitational system is characterized by one parameter, mass or virial temperature, one can find a set of scaling relations among mass, size, X-ray luminosity, temperature, and Compton parameter of Sunyaev-Zel'dovich (SZ) effect if the velocity and mass density fields of baryon fluid in clusters are assumed to be similar to the virialized dark matter halos (Kaiser, 1986). Observed data of galaxy clusters did yield scaling relations (Edge \& Stewart, 1991; David et al., 1993; Wu et al., 1999; Helsdon \& Ponman, 2000; Xue \& Wu, 2000; Croston et al., 2005). However, observed scaling relations generally do not support the predictions given by the baryon-dark matter similarity of virialized dark halos (Helsdon \& Ponman, 2000; Lloyd-Davies et al., 2000).

Since Newtonian gravity is scale-free, the self gravitational system of collisionless dark matter shows scaling behavior if the power spectrum of initial density perturbations is scale-free. These scaling is regardless of whether the underlying gravitational field is virialized (Peebles, 1980). Thus, if the velocity and mass density fields of cosmic baryon matter are given by a similar mapping of the fields of dark matter, one may expect the scaling relations of clusters. However, the similar mapping assumption is correct only in linear regime (Bi et al., 1992), but is baseless in nonlinear regime (Shandarin \& Zeldovich, 1989). The nonlinear evolution of cosmic baryon fluid leads to statistically decouple of the fluid from dark matter. The statistical properties of the velocity and mass density fields of baryon fluid do show deviation from the underlying dark matter field (Pando et al., 2004; He et al., 2005; Kim et al., 2005).

Nevertheless, it has been pointed out by Shandarin \& Zeldovich (1989): the dynamics of cosmic baryon fluid in the expanding universe is scale-free, i.e. no preferred special scales can be identified in the range from the onset of nonlinear evolution down to the length scale of dissipation. It likes fully developed

\footnotetext{
* Corresponding author.

Email addresses: yuanq@mail.ihep.ac.cn (Qiang Yuan), tjzhang@bnu.edu.cn (Tong-Jie Zhang), fanglz@physics.arizona.edu (Li-Zhi Fang).
} 
turbulence in inertial range. This idea recently received substantial developments. With the hydrodynamic simulation sample of the concordance $\Lambda$ CDM model, the velocity field of cosmic baryon fluid is found to be extremely well described by She-Leveque's (SL) scaling formula (She \& Leveque, 1994) in the "inertial range" (He et al., 2006). The SL formula is considered to be the basic statistical features of the scale-free evolution of fully developed turbulence. Moreover, the SL formula comes from self-similar log-Poisson hierarchy, which is related to the hidden symmetry of the Navier-Stokes equations (Dubrulle, 1994; She \& Waymire, 1995). Very recently, it has been shown that the clustering of the mass density field of the cosmic baryon fluid can indeed be well described by a log-Poisson hierarchical cascade (Liu \& Fang, 2008). All the scaling relations and non-Gaussian features predicted from the log-Poisson hierarchy are in very good agreement with the hydrodynamic simulation samples.

These results indicate that, in the scale-free range, the nonlinear evolution of cosmic baryon fluid reaches a statistically quasi-steady state similar to a fully developed turbulence. For turbulence of incompressible fluid, the fluid undergoes a self-similar hierarchical evolution from largest to the smallest eddies and finally dissipates into thermal motion. For cosmic baryon fluid, the clustering on different scales can also be described by a self-similar hierarchy, and the fluid finally falls and dissipates into thermal motion.

This scenario motives us to investigate the scaling relations of clusters from the self-similar hierarchy of cosmic baryon fluid. If the observed scaling relations come from the self-similar hierarchy, one can expect that 1.) the relations of dimensional quantities should be scale-free, i.e. all the scale-dependent coefficients of the scaling relations are scale-invariant; 2.) the relations should be held only if the scales of considered regions are larger than Jeans length, regardless of whether the underlying gravitational field is virialized, i.e. the relations given by cells containing collapsed objects is also available for cells without collapsed objects, only if the scale of cells is larger than the dissipation scale.

Other relevant motivation comes from the non-gravitational heating of baryon gas of clusters. In order to solve the deviation from the similarity of virialized dark halos, various models of non-gravitational heating and cooling of baryonic gas have been proposed (e.g. Valageas \& Silk, 1999; Tozzi \& Norman, 2001; Voit et al., 2002; Zhang \& Pen, 2003; Xue \& Wu, 2003; Nagai et al., 2007). Since these cooling and heating may introduce characteristic scales, the selfsimilar hierarchy will no longer work on these characteristic scales. Therefore, it would be worth to detect the scale on which the above-mentioned two predictions to be broken.

We study these properties with the relation between the Compton parameter 
$y$ of SZ effect and X-ray luminosity $L_{\mathrm{x}}$. The thermal SZ effect is due to the inverse Compton scattering of cosmic microwave background (CMB) photons by hot electrons of baryon fluid. The Compton parameter $y$ depends on the pressure of electron gas (Zeldovich \& Sunyaev, 1969; Sunvaev \& Zeldovich, 1980). There are many works on the $y-L_{x}$ relation (e.g. da Silva et al., 2004; Maughan, 2007; Bonamente et al., 2007). We will, however, focus on the abovementioned two points, which have not yet been addressed right now.

The outline of this paper is as follows. $\S 2$ presents the scaling relations $y=$ $10^{A(r)} L_{x}^{\alpha(r)}$ with observed samples, and shows that $A(r)$ and $\alpha(r)$ are scaleinvariant. $\S 3$ describes the hydrodynamic cosmological simulation samples. The comparison of the scaling relations of simulation samples with observed results is presented in $\S 4$. The conclusions and discussion are given in $\S 5$.

\section{$2 y-L_{\mathrm{x}}$ Scaling Relations from Observed Samples}

\subsection{Data}

To study the scale free properties, we should find the $y(r)-L_{\mathrm{x}}(r)$ relations, where $y(r)$ and $L_{\mathrm{x}}(r)$ are, respectively, the Compton parameter of SZ effect and X-ray luminosity measured from regions with spatial scale $r$. The data of X-ray luminosity of these clusters are taken from McCarthy et al. (2003) (Xray1) and Morandi et al. (2007) (Xray2). The X-ray luminosity from area on comoving scale $r$ is calculated by

$$
L_{\mathrm{x}}(r)=\int_{0}^{\theta_{\mathrm{r}}} L_{x}(\theta) \theta \mathrm{d} \theta,
$$

where $\theta_{\mathrm{r}}=r /\left[(1+z) d_{A}(z)\right]$ is the angular radius corresponding to the comoving scale $r$, and $d_{A}(z)$ is angular diameter distance. $L_{x}(\theta)$ is proportional to the X-ray surface brightness $S_{x}(\theta)$, which can be well fitted by $\beta$-model $S_{x}(\theta)=$ $S_{X 0}\left[1+\left(\theta / \theta_{\mathrm{c}}\right)^{2}\right]^{(1-6 \beta) / 2}$ up to $\theta \sim 10$ arcmin.

Similarly, The mean of $y$ within a region on comoving scale $r$ is given by

$$
y(r)=\frac{2}{\theta_{\mathrm{r}}^{2}} \int_{0}^{\theta_{\mathrm{r}}} y(\theta) \theta \mathrm{d} \theta .
$$

We will use the SZ effect data from Reese et al. (2002) (SZ1) and Bonamente et al. (2006) (SZ2). The former compiled SZ effects of 18 clusters of galaxies span- 


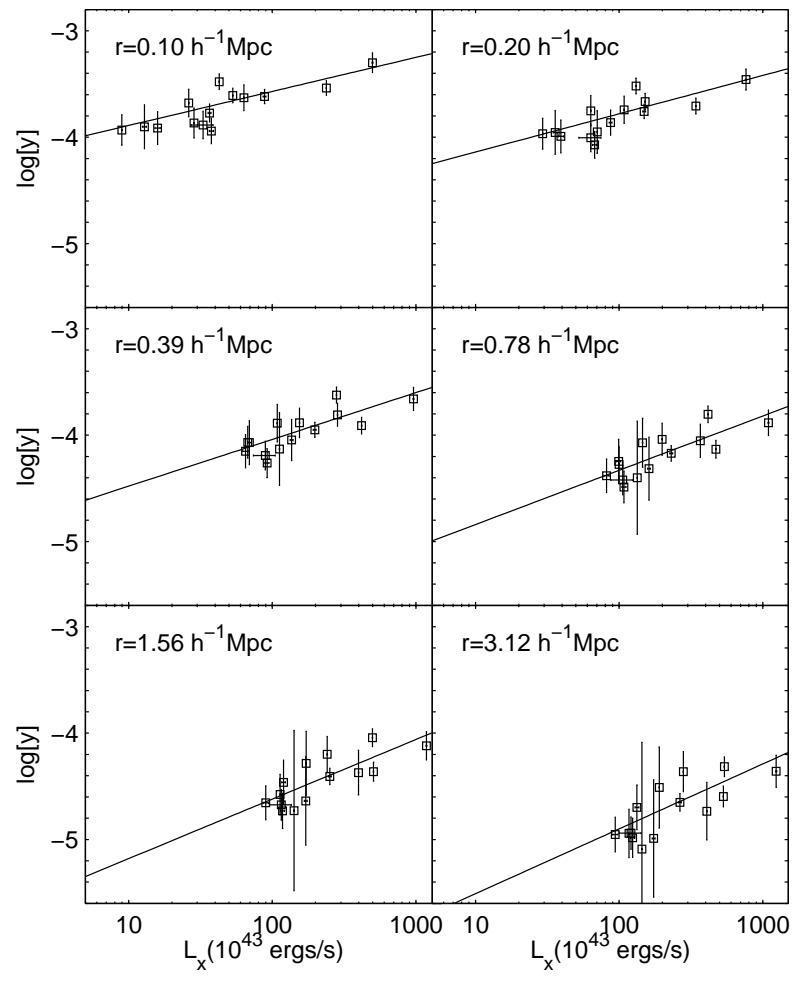

Fig. 1. $y$ - $L_{\mathrm{x}}$ relation of observational samples SZ1+Xray1 (see Table 1). The Compton parameter $y$ are given by average over areas on the comving sizes $0.10,0.20,0.39$, $0.78,1.56$, and $3.12 h^{-1} \mathrm{Mpc}$, respectively. The solid lines indicate the best-fitting for all observational samples.

ning the redshift range of $0.14<z<0.78$, and the later includes 38 clusters in the same redshift range. These data are on angular scales up to $\sim 2$ arcmin, of which the corresponded $r$ is on about the same scale as, or larger than, the Jeans length on redshift $\sim 0.5$. Moreover, the $\theta$-dependencies of $y(\theta)$ are well fitted by $\beta$-model $y(\theta)=y_{0}\left[1+\left(\theta / \theta_{\mathrm{c}}\right)^{2}\right]^{(1-3 \beta) / 2}$. Therefore, it would be reasonable to use the $\beta$ model fitted $y(r)$ to study the $y(r)-L_{\mathrm{x}}(r)$ relation. We will check this point below.

\subsection{Result}

Figure 1 plots the relation of $y(r)$ vs. $L_{\mathrm{x}}(r)$ on scales $r=0.1,0.2,0.39,0.78,1.56$ and $3.12 h^{-1} \mathrm{Mpc}$ respectively. In this figure the SZ and X-ray data are taken from SZ1 and Xray1, respectively. The cluster A370 is excluded as it shows a 3- $\sigma$ discrepancy with the distance-redshift relation (Reese et al., 2002). Three clusters, Cl0016, A611 and A697, are also excluded due to lacking the data of X-ray luminosity, and after all, there are totally 14 clusters used in Figure 1. 
Table 1. $r$-dependence of $\alpha(r)$ and $A(r)$

\begin{tabular}{ccc}
\hline \hline$r\left(h^{-1}\right) \mathrm{Mpc}$ & $\alpha$ & $A$ \\
\hline 0.10 & $0.32 \pm 0.06$ & $-4.21 \pm 0.11$ \\
0.20 & $0.36 \pm 0.08$ & $-4.50 \pm 0.17$ \\
0.39 & $0.44 \pm 0.10$ & $-4.92 \pm 0.23$ \\
0.78 & $0.51 \pm 0.11$ & $-5.35 \pm 0.27$ \\
1.56 & $0.56 \pm 0.12$ & $-5.74 \pm 0.30$ \\
3.12 & $0.61 \pm 0.13$ & $-6.12 \pm 0.33$ \\
\hline
\end{tabular}

We make a best-fitting of the $y(r)-L_{\mathrm{x}}(r)$ relation with a power law $y=$ $10^{A(r)} L_{\mathrm{x}}^{\alpha(r)}$ for various scales $r$ as displayed in Figure 1. The coefficients $\alpha(r)$ and $A(r)$ are listed in Table 1. Both $\alpha(r)$ and $A(r)$ are significantly dependent on the scale $r$. The exponent $\alpha(r)$ increases with scale $r$, while amplitude $A(r)$ decreases with $r$. If the system is given by a similar mapping of virialized halos, the scaling relation should be $y \propto L_{x}^{3 / 4}$ (Cole \& Kaiser, 1988), which means that $\alpha$ is scale-independent and equal to 0.75 . Table 1 shows that the values of $\alpha$ on all scales are less than 0.75 . Accordingly, the baryon fluid on those scales should dynamically deviate from a similar mapping of underlying virialized gravitational field of dark matter halos. To check the effect of the angular scales of $\sim 2 \operatorname{arcmin}(\S 2.1)$, we re-calculate the $y(r)-L_{\mathrm{x}}(r)$ relation with clusters having $d_{A}>1000 \mathrm{Mpc}$, which with $r>0.5 \mathrm{Mpc}$ for angular scales 2 arcmin. We find that the coefficients $\alpha(r)$ and $A(r)$ are consistent with Table 1 within $1-\sigma$ range.

Figure 2 shows $\alpha(r)$ and $A(r)$ as functions of $r$. Note, both $\alpha(r)$ and $A(r)$ can be well fitted, respectively by $a_{\alpha}+b_{\alpha} \log r$ and $a_{A}+b_{A} \log r$, and therefore, both $\alpha(r)$ and $A(r)$ are scale-invariant. The amplitude $10^{A(r)}$ actually is a power law of $r$. This result is consistent with the dynamics of self-similar hierarchy. To further test this result, we used other data sets of SZ effect and X-ray luminosity. The results are also shown in Figure 2. Although these data sets are generally different from each other, all results of $\alpha(r)$ and $A(r)$ can well be fitted by the straight line of $\log r$. The fitting parameters are listed in Table 2. They are the same within 1- $\sigma$ errors. It strongly supports the scenario of scale-free dynamics. 


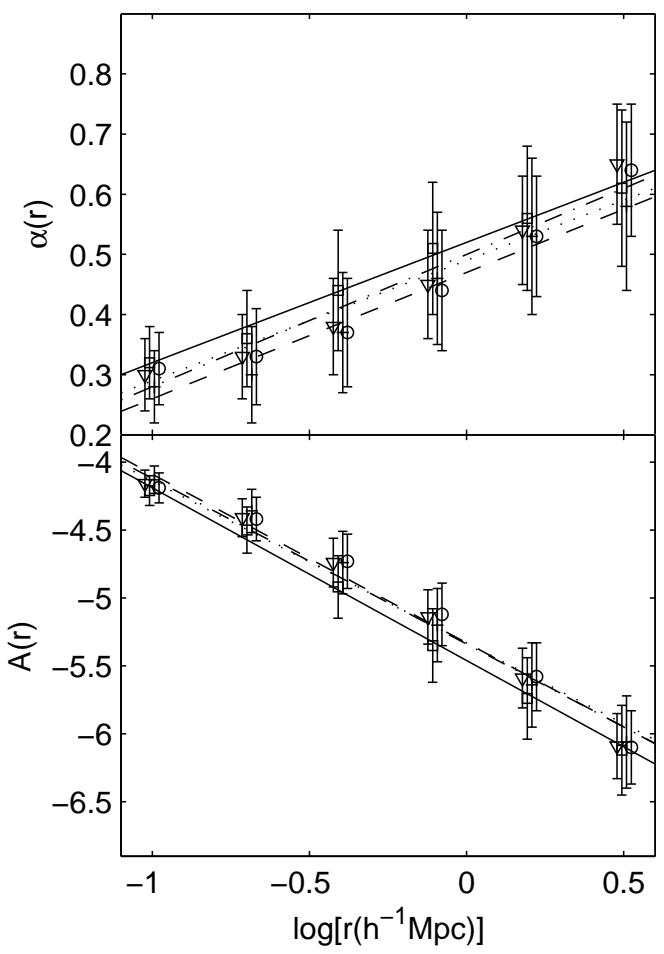

Fig. 2. The scale-dependence of the coefficients $\alpha(L)$ and $A(r)$ for various observational samples: SZ1+Xray1 (solid); SZ1+Xray2 (dashed); SZ2+Xray1 (dotted); and SZ2+Xray2 (dot-dashed).

Table 2. Fitting Results of $\alpha(r)$ and $A(r)$.

\begin{tabular}{ccccc}
\hline \hline sample $^{1}$ & $a_{\alpha}$ & $b_{\alpha}$ & $a_{A}$ & $b_{A}$ \\
\hline SZ1+Xray1 & $0.52 \pm 0.05$ & $0.20 \pm 0.07$ & $-5.46 \pm 0.13$ & $-1.27 \pm 0.17$ \\
SZ1+Xray2 & $0.47 \pm 0.06$ & $0.21 \pm 0.08$ & $-5.33 \pm 0.13$ & $-1.24 \pm 0.17$ \\
SZ2+Xray1 & $0.49 \pm 0.05$ & $0.20 \pm 0.07$ & $-5.33 \pm 0.11$ & $-1.19 \pm 0.15$ \\
SZ2+Xray2 & $0.50 \pm 0.04$ & $0.22 \pm 0.06$ & $-5.34 \pm 0.10$ & $-1.22 \pm 0.13$ \\
\hline
\end{tabular}

1 Z1 and SZ2 from Reese et al. (2002) and Bonamente et al. (2006), Xray1 and Xrya2 from McCarthv et al. (2003) (Xray1) and Morandi et al. (2007), respectively

It should also be pointed out that the scales of $r \leq 0.39 h^{-1}$ Mpc are actually less than the Jeans length of baryon fluid. However, the coefficients $\alpha(r)$ and $A(r)$ are still following the self-similar straight lines. In addition, $\alpha(r)$ is seen to be less than the value 0.75 from virialized halos. It implies that the dynamics 
of baryon fluid on these scales seems still to be scale free.

\section{SZ effect samples of cosmological hydrodynamical simulations}

\subsection{Simulation}

Before embarking on the numerical calculations, we give a brief summary on the baryon fluid when it is in the turbulence-like or self-similar hierarchical clustering. The dynamics of growth modes of clustering in an expanding universe is sketched by a stochastic force driven Burgers equation (Berera \& Fang, 1994; Jones, 1999; Matarrese \& Mohavaee, 2002). The turbulence-like behavior is due to Burgers' turbulence, which will be developed when the Burgers Reynolds number is large (Polyakov, 1995; Boldyrev et al., 2004; Kraichnan, 1968; Lässig, 2000). A turbulent flow in incompressible fluid consists of vortexes, while the clustering of cosmic matter is irrotational, because the modes with vorticity of the perturbed mass density field do not grow. The Burgers' turbulence made the initially random field to result in a collection of shocks and a smooth variation of the field between the shocks. For cosmic baryon fluid, the Burgers Reynolds number generally is larger in nonlinear regime (He et al., 2004). Therefore, the baryon fluid in nonlinear regime consists of a collection of shocks with various strengths in both high, moderate and even low density areas. The kinetic energy of fluid is dissipated due to the shocks on various scales. The kinetic energy of fluid is effectively converted into ther-

mal energy (He et al., 2006). In this context, it is clear that the method of hydrodynamical simulations should be capable of capturing discontinuities, like shocks, and their effects on energy conversion precisely.

We will use the Weightly Essentially NonOscillatory (WENO) algorithm, which is effective to capture shocks and other discontinuities of the baryon fluid, and to give precise value of the fluid field between the discontinuities (Feng et al., 2004). This algorithm has been tested with 1.) Shock tube; 2.) the Sedov-Taylor self-similar blast wave solution, or the Bertschinger's similarity solution; 3.) Zeldovich pancake. It is effectively to produce the baryon mass density contour, baryon temperature contour around massive halos (Feng et al., 2004; He et al., 2004, 2005). Some other properties of this simulation algorithm can also be found in Feng et al. (2004) and He et al. (2004).

The simulations are performed in a cubic box $100^{3} h^{-3} \mathrm{Mpc}^{3}$ with a $1024^{3}$ grid, and the number of dark matter particles is $512^{3}$. The mass of the dark matter particle is $\sim 10^{9} \mathrm{M}_{\odot}$, which corresponds to a density resolution about 0.01 times of the mean density of intergalactic medium (IGM). The grid size 
is then $100 / 1024 \sim 0.10 h^{-1}$ Mpc. This scale is smaller than the Jeans length at $z \leq 1$ (Bi et al., 2003). Therefore, the sample is suitable to describe the baryon fluid from the dissipation scale to a few ten $h^{-1}$ Mpc. We use the concordance $\Lambda$ CDM cosmology model with parameters $\Omega_{\mathrm{m}}=0.27, \Omega_{\mathrm{b}}=0.044$, $\Omega_{\Lambda}=0.73, h=0.71, \sigma_{8}=0.84$, and spectral index $n=1$ and the ratio of specific heats is $\gamma=5 / 3$. The transfer function is calculated using CMBFAST (Seljak \& Zaldarriaga, 1996).

The atomic processes including ionization, cooling and heating are modeled as the method in Theuns et al. (1998). We take a primordial composition of $\mathrm{H}$ and $\mathrm{He}(X=0.76, Y=0.24)$ and use an ionizing background model of Haardt \& Madau (2001).

The simulations start at the redshift $z=99$, and output the density, velocity and temperature fields at redshifts $z=2,1,0.5$, and 0 . It is easy for hydrodynamic simulation with Eulerian variable to reach low-density regions. They are suitable for a uniform analysis of weakly as well as strongly clustered fields of baryon fluid. These samples have been successfully applied to reveal the self-similar hierarchical behavior of cosmic baryon fluid (Kim et al., 2005; He et al., 2006; Liu \& Fang, 2008), to explain the transmitted flux of HI and HeII Ly $\alpha$ absorption of quasars (Liu et al., 2006), and to study the relations between X-ray luminosity and temperature of groups of galaxies (Zhang et al., 2006).

As mentioned in $\S 1$, the self-similar hierarchical scenario predicts that the relation $y(r)=10^{A(r)}\left[L_{\mathrm{x}}(r)\right]^{\alpha(r)}$ given by areas containing collapsed objects should also be available for areas without collapsed objects, only if $r$ is larger than the dissipation scale. On the other hand, either the effects of heating by injecting hot gas or metal cooling are localized in massive halos. Their characteristic scales are less than the Jeans length. Therefore, one can expect that the $y-L$ relations given by observed samples should be the same as that given by simulation samples either with or without considering the localized heating and cooling processes. To consider the effect of the metal abundance, we also use a sample with metal cooling of Zhang et al. (2006). For this sample, The metal cooling and metal line emission is calculated by the phenomenological method: 1) assuming an uniform evolving metallicity $Z=0.3 Z_{\odot}\left(t / t_{0}\right), t_{0}$ being the present universe age; 2 ) computing the cooling function using the table of Sutherland \& Dopita (1993).

\subsection{Samples}

When relativistic corrections is negligible, the Compton parameter $y$ of the thermal SZ effect along a line of sight, $l$, with an angular distance $\theta$ from the 
center of a cluster in the plane of the sky is given by

$$
y(\theta)=\frac{k_{\mathrm{B}} \sigma_{\mathrm{T}}}{m_{\mathrm{e}} c^{2}} \int n_{\mathrm{e}}(l, \theta) T_{\mathrm{e}}(l, \theta) \mathrm{d} l
$$

where $\sigma_{\mathrm{T}}$ is the cross section of the Thomson scattering; $n_{\mathrm{e}}$ and $T_{\mathrm{e}}$ are, respectively, the number density and temperature of hot electrons. Since $\mathrm{H}$ and He atoms are almost fully ionized, we take the electron density $n_{\mathrm{e}}=\rho / \mu_{\mathrm{e}} m_{\mathrm{p}}$ where $\rho$ is the density of baryon gas, $\mu_{\mathrm{e}}=2 /(1+X)$ with a hydrogen abundance of $X=0.76$.

Using simulated density and temperature fields, we calculate the parameter $y(\theta)$ with Eq. (3). The mean of parameter $y(r)$ on various scales can be obtained using the scaling function of the discrete wavelet transform (DWT)

$$
y_{\mathbf{j}, \mathbf{l}}=\frac{1}{\int \phi_{\mathbf{j}, 1}(\mathbf{x}) \mathrm{d} \mathbf{x}} \int y(\mathbf{x}) \phi_{\mathbf{j}, \mathbf{l}}(\mathbf{x}) \mathrm{d} \mathbf{x}
$$

where $\phi_{\mathbf{j}, \mathbf{l}}(\mathbf{x})$ is the scaling functions related to cell $(\mathbf{j}, \mathbf{l})$ with comoving size $100 / 2^{j} h^{-1} \mathrm{Mpc}$ and at position $\mathbf{l}=\left(l_{1}, l_{2}, l_{3}\right)$. The details of the DWT analysis can be found in Fang \& Thews (1998). We take the comoving size $100 / 2^{j} h^{-1}$ Mpc with $j=10,9,8,7,6$ and 5, corresponding to scales $0.10,0.20,0.39,0.78$, 1.56 and $3.12 \mathrm{~h}^{-1} \mathrm{Mpc}$, which are the same as that used in the analysis of the observed samples in last section.

The total X-ray luminosity of thermal bremsstrahlung emission from a cell $(\mathbf{j}, \mathbf{l})$ is calculated by the same way as Zhang et al. (2006)

$$
\left(L_{\mathbf{x}}\right)_{\mathbf{j}, \mathbf{l}}=V_{j} \frac{1}{\int \phi_{j, l}(\mathbf{x}) \mathrm{d} \mathbf{x}} \int \epsilon^{f f}(\mathbf{x}) \phi_{j, l}(\mathbf{x}) \mathrm{d} \mathbf{x}
$$

where $\epsilon^{f f}(\mathbf{x})$ is the map of X-ray emissivity, and $V_{j}$ is the volume of cell $(\mathbf{j}, \mathbf{l})$.

The DWT decomposition has following advantages. First, the set of scaling functions are orthogonal and complete, and therefore, the decomposition of Eqs.(3) and (4) does not give rise to false correlation. Second, the DWT cell $(\mathbf{j}, \mathbf{l})$ with high mass density can directly be used to identify clumpy structures. The DWT-identified cells on scale $1.5 h^{-1} \mathrm{Mpc}$ is statistically the same as clusters identified by traditional method, such as the friend-of-friend algorithm (Xu et al., 1998). Therefore, the DWT variables $y_{\mathbf{j}, 1}$ and $\left(L_{\mathbf{x}}\right)_{\mathbf{j}, 1}$ provides a uniform description of the Compton parameter and X-ray luminosity of the whole field, regardless of the dynamical details of all cells $(\mathbf{j}, \mathbf{l})$. Third, the DWT variables can be applied directly to non-Gaussian behavior (He et al., 2006; Liu \& Fang, 2008) We will use the Harr wavelet (Daubechies 2) to do the 


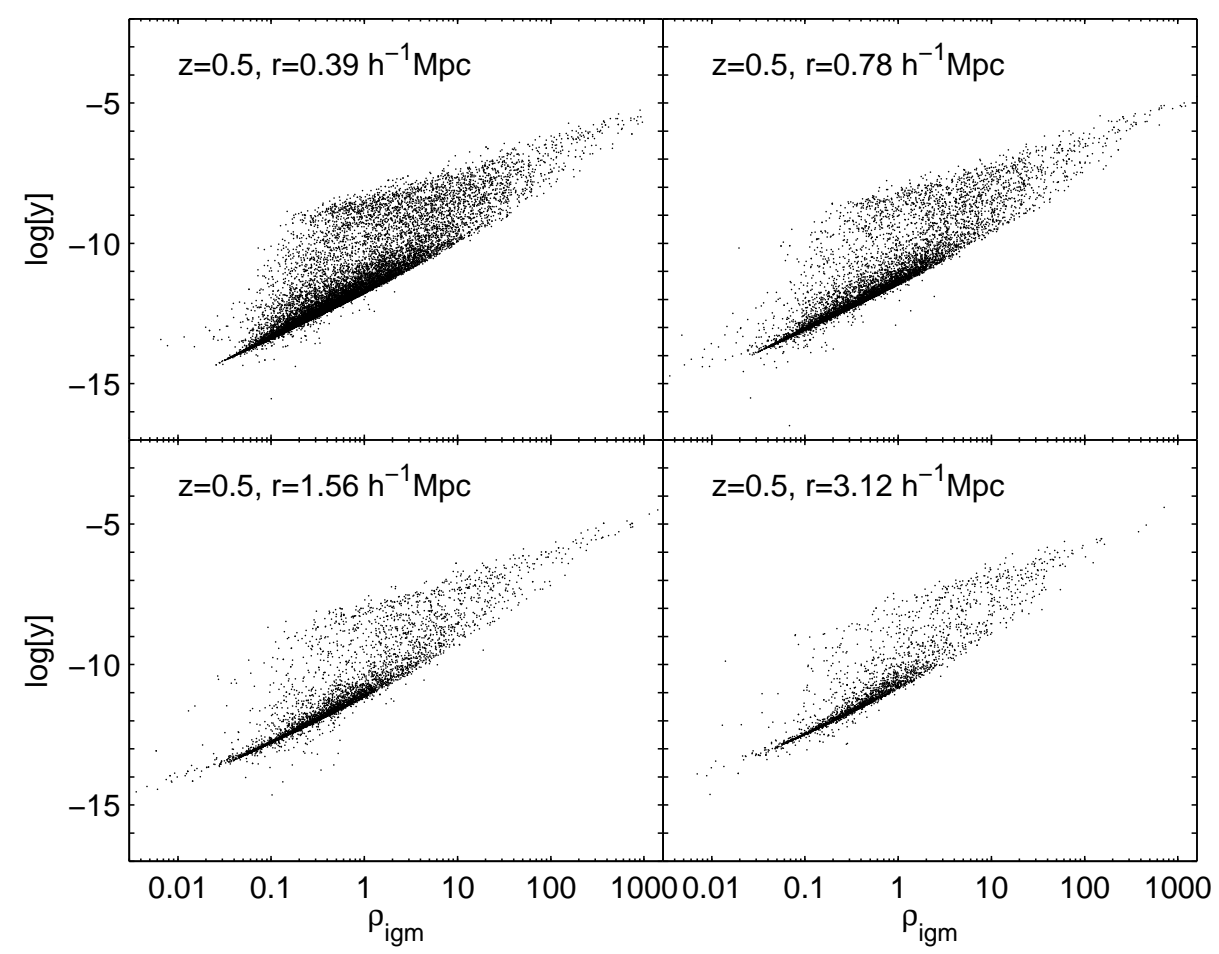

Fig. 3. Compton parameter $y$ vs. baryon density $\rho_{\text {igm }}$ of simulation samples at redshift $z=0.5$, in which $y$ and $\rho_{\text {igm }}$ are the mean over cells with comoving scales $r=0.39,0.78,1.56$, and $3.12 h^{-1} \mathrm{Mpc}$, respectively. $\rho_{\text {igm }}$ is in unit of mean mass density $\bar{\rho}_{\text {igm }}$ of the field.

calculation below. We also repeat the calculations with wavelet Daubechies 4 . The statistical features given by Daubechies 4 are basically the same as Haar wavelet.

\section{Scaling Relations between the SZ Effect and X-ray Luminosity}

\section{$4.1 y-\rho_{\mathrm{igm}}$ and $L_{x}-\rho_{\mathrm{igm}}$ relations}

Figure 3 shows the relations between the mean Compton parameter $y(r)$ and mean mass density $\rho_{\text {igm }}(r)$ of cells on scale $r$ for the simulation samples at redshift $z=0.5$. The comoving scales $r$ are taken to be $0.39,0.78,1.56$ and $3.12 h^{-1} \mathrm{Mpc}$ respectively. Figure 3 has a dark area as a bottom envelop of the $y$ - $\rho_{\text {igm }}$ distribution. It gives a tight correlation between $y$ and $\rho_{\text {igm }}$ and can be described approximately by a power law of $y \propto \rho_{\text {igm }}^{1.8}$ for all scales $r$. This relation is basically consistent with the so-called adiabatic 'equation of state' $T \propto \rho^{2 / 3}$ if considering $y \propto \rho_{\mathrm{igm}} T$. 


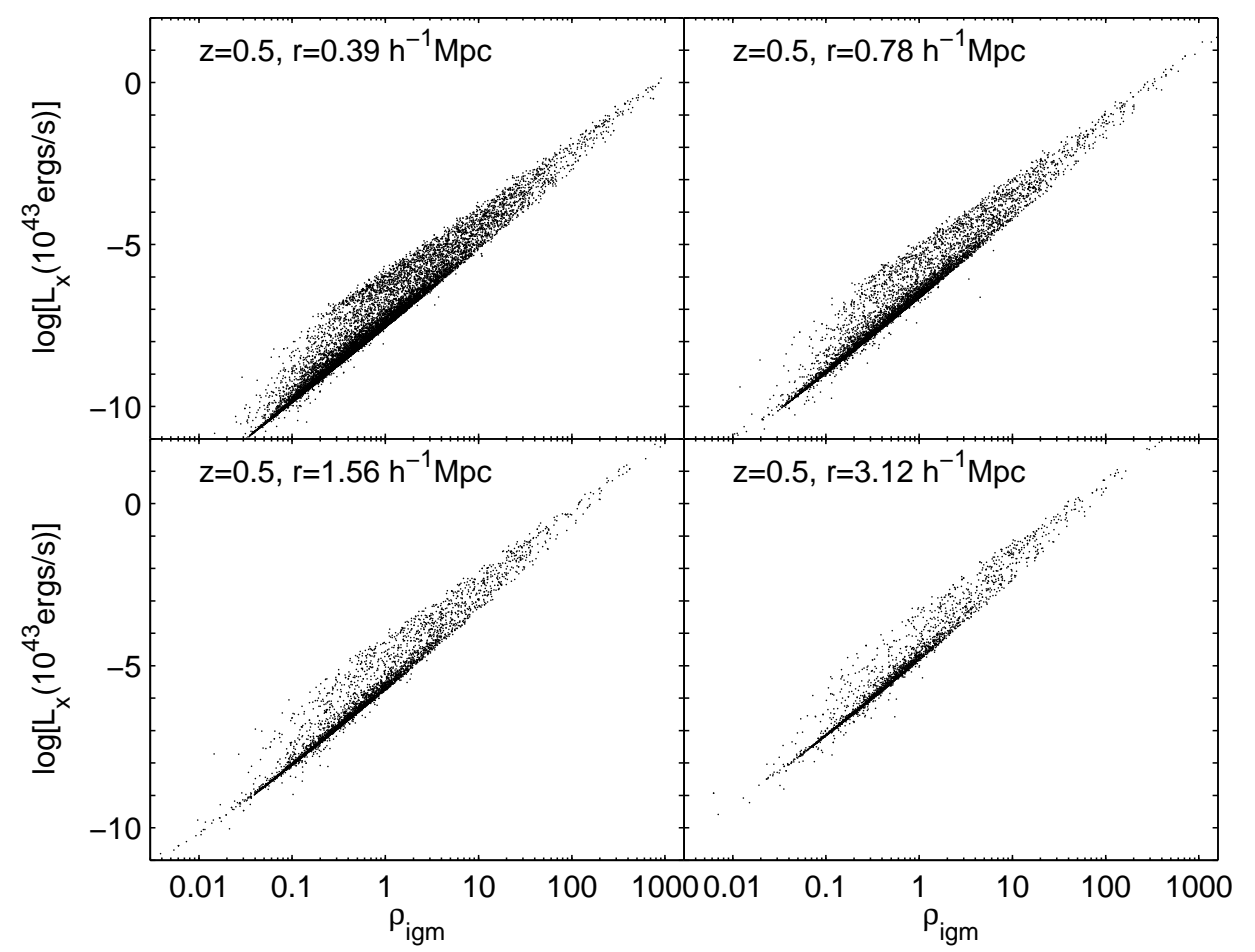

Fig. 4. X-ray luminosity $L_{x}$ vs. baryon density $\rho_{\text {igm }}$ of simulation samples at redshift $z=0.5$. The $L_{x}$ is the total X-ray luminosity from cell with comoving scale $r=0.39$, $0.78,1.56$, and $3.12 h^{-1} \mathrm{Mpc}$. $\rho_{\text {igm }}$ are the mean of density within the cell. $\rho_{\text {igm }}$ is in unit of mean mass density $\bar{\rho}_{\text {igm }}$ of the whole field.

In Figure 4, the similar analysis has been performed for the relations between the total X-ray luminosity $L_{x}(r)$ and mean mass density $\rho_{\text {igm }}(r)$. Clearly, there is also a dark area as the bottom envelop of the $L_{X}-\rho_{\text {igm }}$ distribution. The tight correlation of the dark area yields $L_{X} \propto \rho_{\text {igm }}^{2.4}$ for all scales. This is expected if considering $L_{X} \propto \rho_{\mathrm{igm}}^{2} T^{1 / 2}$.

Therefore, $y$ and $L_{X}$ given by the tight correlations of bottom envelops in Figures 3 and 4 imply $y \propto L^{0.75}$, which is the same as that given by a virialized underlying gravitational field. If we adopt the adiabatic 'equation of state' $T \propto$ $\rho^{2 / 3}$, the $y-L_{\mathrm{x}}$ scaling relation should be $y \propto L_{x}^{0.71}$. The observed coefficient $\alpha$ are less than 0.75 on all scales. The reason for $\alpha<0.75$ is clearly shown by Figures 3 and 4 . For a given $\rho_{\mathrm{igm}}$, the distribution of $y$ is significantly scattered from the bottom envelop. For sample at $z \simeq 0.5$, the data points of $y$ corresponding to $\rho_{\mathrm{igm}} \simeq 1$ scatter in the range from $\sim 10^{-11}$ to $10^{-8}$. The scattering is due to the heating of Burgers' shocks, which leads to the baryon fluid to be multiphasic, i.e. the relation between temperature and mass density can not be described by one polytropic equation. The points of $y$ higher than the bottom envelop correspond to state with temperature higher than those given by the 'equation of state' $T \propto \rho^{2 / 3}$ (He et al., 2004; Kim et al., 2005). 


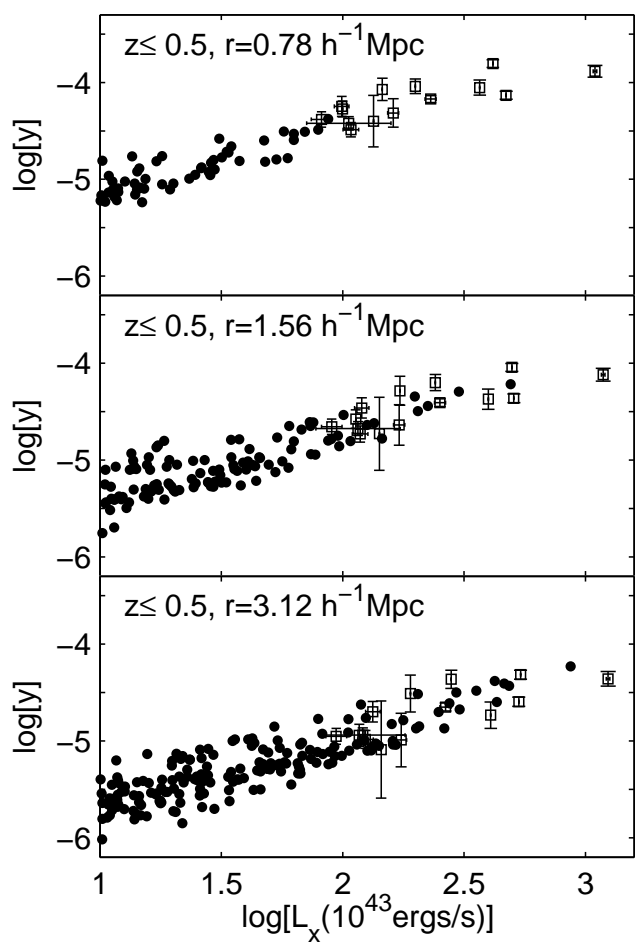

Fig. 5. The scaling relation between the Compton parameter $y(r)$ and X-ray luminosity $L_{\mathrm{X}}(r)$. The scales $r$ are $0.78,1.56$, and $3.12 h^{-1} \mathrm{Mpc}$. The observed data points (squares with errorbars) are from SZ1+Xray1 and the simulation data points (dotted) are taken from sample of redshift $z \leq 0.5$.

The multiphases of baryon fluid are seen for all scales. The scattering in the $y$ $\rho_{\text {igm }}$ distribution is substantial in high mass density areas $\left(\rho_{\mathrm{igm}}>1\right)$ as well as low mass density areas $\left(\rho_{\text {igm }}<1\right)$. The $L_{x}-\rho_{\text {igm }}$ distribution (Figure 4$)$ shows similar scattering. It can be understood that the Burgers' shock heating is not only working in high density regions, but also in low density areas. It leads to the deviation of $\alpha$ from 0.75 .

\section{2 $y$ - $L_{\mathrm{x}}$ scaling relation}

We now study the scaling relation between $y(r)$ and $L_{\mathrm{x}}(r)$. Figure 5 plots the distribution of the Compton parameter $y(r)$ against the X-ray luminosity $L_{\mathrm{x}}(r)$ for the simulation samples at $z \leq 0.5$, in which the comoving scales of the cell are taken to be $0.78,1.56$ and $3.12 h^{-1} \mathrm{Mpc}$ respectively. The observed points of $y(r)-L_{\mathrm{x}}(r)$ are taken from the samples SZ1+Xray1.

On the scale of $3.12 h^{-1} \mathrm{Mpc}$, the simulation data can be fitted by observations very well. Using the simulation data with $\log \left[L_{X}(r) / 10^{43} \mathrm{erg} / \mathrm{s}\right] \geq 1$, we found 
that the best fitting scaling relation $y=10^{A} L_{x}^{\alpha}$ of simulation sample gives $\alpha=0.65 \pm 0.03$ and $A=-6.31 \pm 0.05$, which are the same as observed samples shown in Table 1. For the scale of $1.56 h^{-1} \mathrm{Mpc}$, Figure 5 also shows the consistency between observed and simulated samples. Since $\alpha$ is less than 0.75, the scaling relation shown in Figure 5 is dominated by data points located above the bottom envelop of Figure 3 . In other word, the $y-L_{\mathrm{x}}$ relations are mainly determined by the structures which are involved in the evolution of the Burgers turbulence.

On scale $0.78 h^{-1} \mathrm{Mpc}$, the simulation sample still shows the same trend as observed data, but there are fewer points with $\log \left[L_{X}(r) / 10^{43} \mathrm{erg} / \mathrm{s}\right] \geq 2$. This phenomenon is more serious on scale $0.39 h^{-1} \mathrm{Mpc}$. In this case, the best fitting scaling relation yields $\alpha=0.65 \pm 0.01$ and $A=-5.47 \pm 0.01$, which deviates from the observed result $\alpha=0.44 \pm 0.10$ and $A=-4.92 \pm 0.23$ (Table 1). The deviation on scales $\leq 0.78 \mathrm{~h}^{-1} \mathrm{Mpc}$ is expected. Since the scale $0.78 h^{-1} \mathrm{Mpc}$ is typical of the so-called $R_{2500}$ of clusters, and it is less than the virialization radius of clusters. Within this scale range, dissipation and non-gravitational processes are involved. It is beyond the regime of scale-free evolution.

Because the observed data contains clusters with redshifts higher than 0.5, we also made a comparison between observed data and simulation samples of $z \leq 1$. The results are displayed in Figure 6, which yields almost the same results as Figure 5. We also analyzed the simulation samples including metal cooling of Zhang et al. (2006). The result is given in Figure 7. It shows the effect of metal cooling does not change the feature of Figure 5. Although metal cooling may have a big effect on $L_{\mathrm{x}}$ for groups. But it will have the similar effect on $y$. The $y$ - $L$ x scaling relation still keeps self-similar in the inertia range.

\subsection{Available range of the $y$ - $L_{\mathrm{x}}$ scaling relation}

As emphasized above, the scaling relation between physical quantities of cosmic baryon fluid due to the Burgers turbulence should hold for the entire field. Those relations inferred from statistical analysis made in regions containing collapsed structures, like clusters, should also be applicable in regions without that structures. Actually, Figures $5-7$ have already shown that the $y-L_{x}$ scaling relation works well for all regions containing strong X-ray emission $\log \left[L_{X}(r) / 10^{43} \mathrm{erg} / \mathrm{s}\right] \geq 1$.

Figure 8 presents the $y$ - $L_{x}$ scaling relation covering a much wider range of the X-ray luminosity $-3 \leq \log \left[L_{X}(r) / 10^{43} \mathrm{erg} / \mathrm{s}\right] \leq 3$, from rich clusters to weakly clustered areas. It shows clearly that the scaling relation of Figure 1 is available in the weakly clustered areas. 


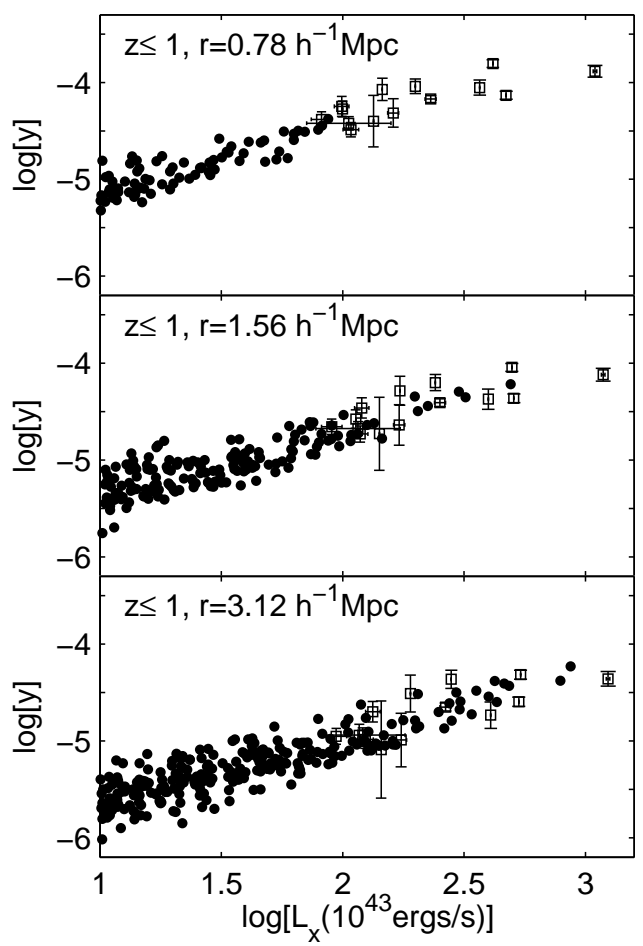

Fig. 6. The scaling relation between the Compton parameter $y(r)$ and X-ray luminosity $L_{\mathrm{X}}(r)$. The scale $r$ are $0.78,1.56$, and $3.12 h^{-1} \mathrm{Mpc}$ respectively. The observed data points ( squares with errorbars) are from SZ1+Xray1. The simulation data points (dots) are taken from sample of redshift $z \leq 1$.

Table 3. Fitting Coefficients $\alpha$ and $A$ for Simulation Data

\begin{tabular}{ccc}
\hline \hline range oflog $\left[L_{X}(r)\right]$ & $\alpha$ & $A$ \\
\hline $1<\log \left[L_{X}(r) / 10^{43} \mathrm{erg} / \mathrm{s}\right]<3$ & $0.65 \pm 0.03$ & $-6.31 \pm 0.05$ \\
$0<\log \left[L_{X}(r) / 10^{43} \mathrm{erg} / \mathrm{s}\right]<3$ & $0.63 \pm 0.01$ & $-6.27 \pm 0.02$ \\
$-1<\log \left[L_{X}(r) / 10^{43} \mathrm{erg} / \mathrm{s}\right]<3$ & $0.65 \pm 0.01$ & $-6.29 \pm 0.01$ \\
$-2<\log \left[L_{X}(r) / 10^{43} \mathrm{erg} / \mathrm{s}\right]<3$ & $0.65 \pm 0.01$ & $-6.30 \pm 0.01$ \\
$-3<\log \left[L_{X}(r) / 10^{43} \mathrm{erg} / \mathrm{s}\right]<3$ & $0.59 \pm 0.00$ & $-6.32 \pm 0.01$ \\
\hline
\end{tabular}

We did a fitting of $y$ - $L_{x}$ scaling relation in the simulation sample on the scale of $r=3.12 h^{-1} \mathrm{Mpc}$ and $z \leq 0.5$ with different ranges of $\log L_{X}(r)$. The bestfitting values of the coefficients $\alpha$ and $A$ for various ranges of $\log L_{X}(r)$ are listed in Table 3. Obviously, the coefficients $\alpha$ and $A$ are almost independent of the range of $\log L_{X}(r)$. The scaling relation, $y(r)=10^{A(r)}\left[L_{X}(r)\right]^{\alpha(r)}$ is very stable within $10^{39}<L_{x}<10^{46} \mathrm{erg} \mathrm{s}^{-1}$. If $y$ and $L_{x}$ are measured from regions on scales larger than dissipation scales, the $y$ - $L_{x}$ scaling relation still holds 


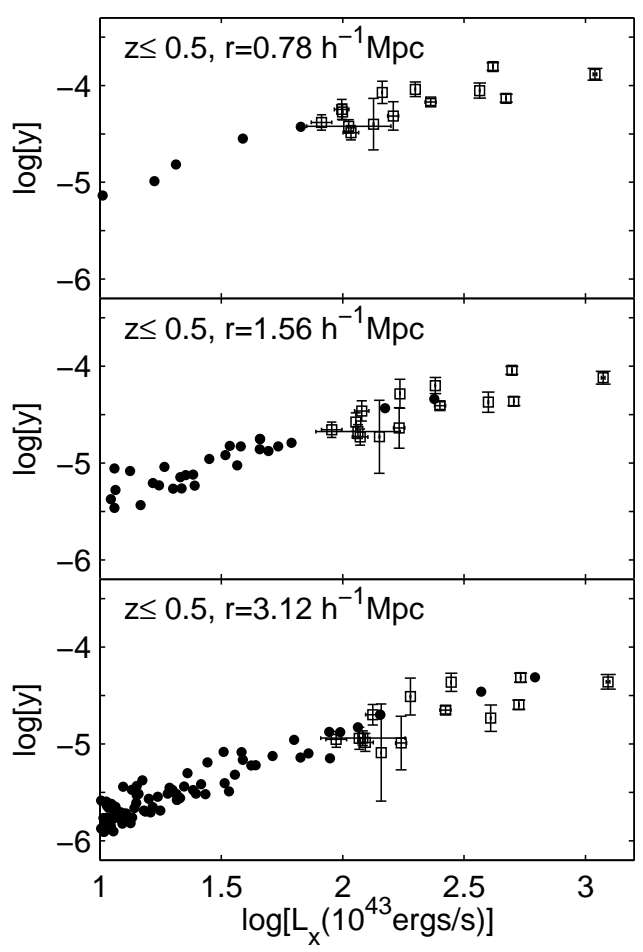

Fig. 7. The same as Figure 5, but with simulation sample in which the the metal cooling and metal line emission are considered.

regardless of the dynamical details on the dissipation scales. The wide range of $L_{\mathrm{x}}$ is consistent with the scenario of self-similar hierarchical evolution, which gives a unified description of the dynamics of clustering on various level in the scale-free range.

Similar analysis was made in simulation samples on scale of $r=1.56 h^{-1} \mathrm{Mpc}$ and $0.78 h^{-1} \mathrm{Mpc}$ at redshifts $z=0,0.5$ and 1 respectively. These scaling relations are also valid within the entire range of $10^{39}<L_{x}<10^{46} \mathrm{erg} \mathrm{s}^{-1}$ as well. Moreover, from Figure 8, we can see that the scaling relations basically are redshift-independent within the range $z \leq 1$. Along with the decreasing redshift, there are more data points with larger $\log L_{X}$. The $y$ - $L_{x}$ correlation shown in Figure 8 can be seen as a tree with root at left-bottom corner, and tip at right-top corner. The formation of clustered objects leads to the growth of the tip of the tree along the direction given by the scaling relation $y(r)=10^{A(r)}\left[L_{x}(r)\right]^{\alpha(r)}$. 


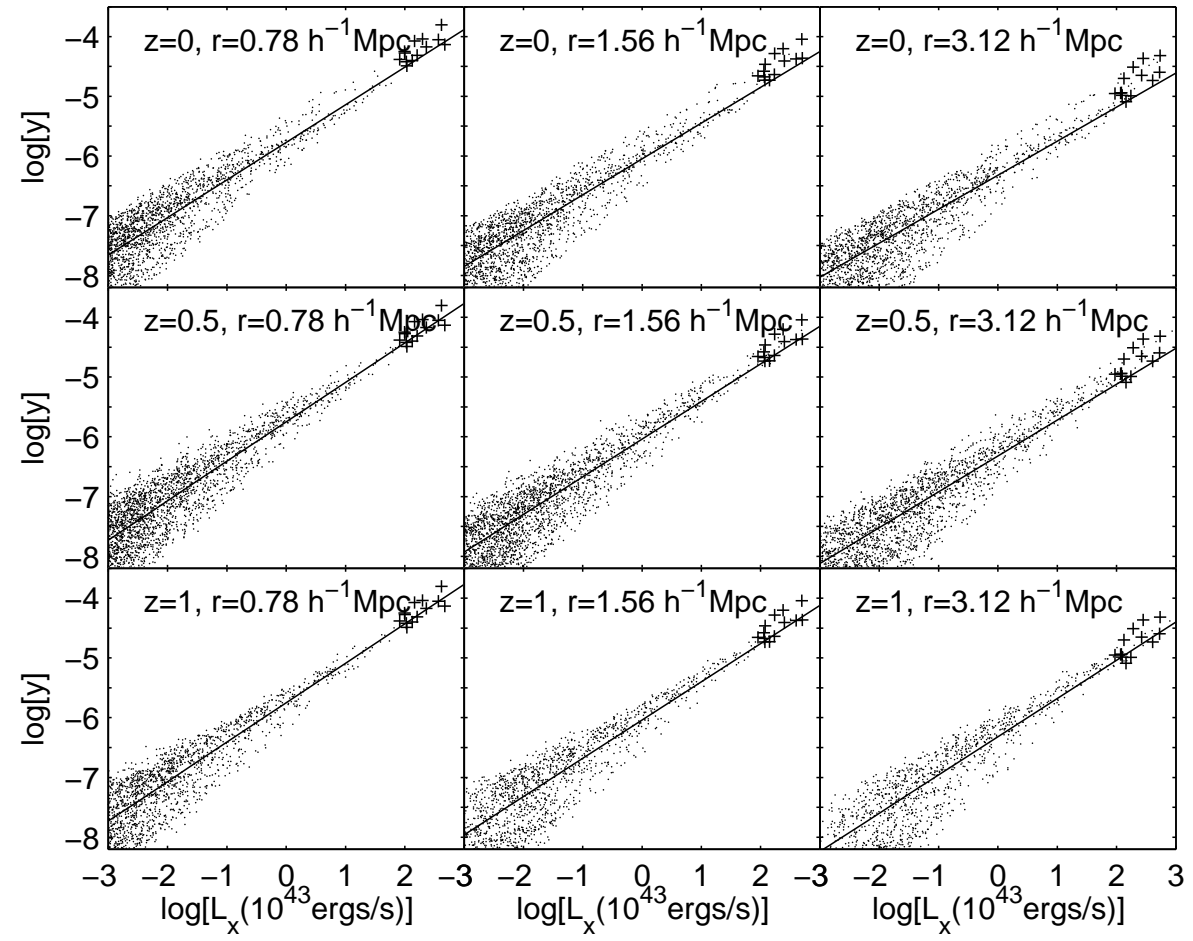

Fig. 8. The scaling relation between the Compton parameter $y$ and X-ray luminosity $L_{\mathrm{x}}$ of simulation data at redshift $z=0$ (top), 0.5 (middle), and 1 (bottom) respectively. The scale $r$ are 0.78 (left), 1.56 (middle) and 3.12 (right) $h^{-1} \mathrm{Mpc}$ respectively. The solid line is given by the fitting of simulation data.

\section{Discussion and Conclusions}

The scaling studied in this paper comes from the scale-free dynamics of cosmic baryon fluid in the expanding universe. That is, there is no preferred special scales can be identified in the range from the onset of nonlinear evolution down to the characteristic length of dissipation. The clustering evolution is described by self-similar hierarchy of baryon fluid. This self-similar hierarchy is different from the gravitational self-similarity of virialzed system, which is characterized by the mass of the system. On the other hand, the scaling from the scale-free dynamics is characterized by the scale range, on which physical quantities of the mass and velocity field of cosmic baryon fluid are measured. That is, the dimensional quantities measured in cells with size larger than the dissipation characteristic length should satisfy the same scaling relations, regardless whether the cells contains massive collapsed objects. The scaling will be broken if the scale is less than the characteristic length of dissipation.

We demonstrated this scaling with the $y(r)-L_{\mathrm{x}}(r)$ relation of baryon field. The observed $y(r)-L_{\mathrm{x}}(r)$ relation can be well reproduced with the hydrodynamical 
simulation. The important point is that the fitting of $y(r)-L_{\mathrm{x}}(r)$ scaling is not based on the identified clusters in simulation samples, but using all cells on a given scale $r$, regardless whether the cell contains rich clusters with strong X-ray emission. In other words, the scaling relations $y(r)=10^{A(r)}\left[L_{\mathrm{x}}(r)\right]^{\alpha(r)}$ can be used to describe cells containing strong X-ray emission, $L_{\mathrm{x}}>10^{43} \mathrm{erg}$ $\mathrm{s}^{-1}$, as well as very weak X-ray emission regions $L_{\mathrm{x}} \sim 10^{40} \mathrm{erg} \mathrm{s}^{-1}$. This result supports the self-similar hierarchical scenario of the clustering of baryon fluid.

The observed $y(r)-L_{\mathrm{x}}(r)$ relations starts to show a deviation from simulation results on scales smaller than $0.78 \mathrm{~h}^{-1} \mathrm{Mpc}$, which would be the characteristic scale of the dissipation. We calculated the Jeans lengths for each objects used in our statistics. All of the Jeans lengths are found to be less than $0.8 \mathrm{~h}^{-1} \mathrm{Mpc}$. It is very well consistent with our result. Moreover, this result is also consistent with previous studies on the non-gravitational heating of clusters. The nongravitational heating generally is considered to be due to the injection of hot gas and energy from SN and AGN. However, the significant effect of injecting hot gas and energy by $\mathrm{SN}$ is mostly arising from dwarf galaxies, i.e. on scales much smaller than clusters. The Ly $\alpha$ observations of protoclusters shows that the feedback of AGN is not strong enough to heat the gas of clusters within comoving size $0.5 \mathrm{~h}^{-1} \mathrm{Mpc}$ (Adelberger et al., 2003). Therefore, the effect of the SN and AGN heating would be dramatic only on scales smaller than about $0.5 \mathrm{~h}^{-1} \mathrm{Mpc}$, but probably cannot heat gas within cells on scales $\geq 1 \mathrm{~h}^{-1} \mathrm{Mpc}$ to amount of the order of $1 \mathrm{keV}$ per nucleon (Pen, 1999; Wu et al., 1999). Actually we find that scaling relations on scales $>0.78 \mathrm{~h}^{-1} \mathrm{Mpc}$ are still held after subtracting the contributions from the central part $\left(<0.2 \mathrm{~h}^{-1} \mathrm{Mpc}\right)$ of clusters to both $L_{\mathrm{x}}$ and $y$, as done in Markevitch (1998); Maughan (2007).

As an application, the scaling relations of $y(r)-L_{\mathrm{x}}(r)$ would be useful for estimating the contamination of SZ effect on CMB, which is important, especially, on small scales (Cao et al., 2006). Recent simulation has shown that the Planck project would be capable of probing $y$ on the order of $y=10^{-7}-10^{-8}$ (Dolag et al., 2005). It might give a direct test on the universal scaling relations of $y(r)-L_{\mathrm{x}}(r)$ given by the self-similar hierarchical evolution.

Our simulation of the baryon fluid is within the Eulerian framework. One can also study the nature of intermittency of fluid with Lagrangian point of view. In this approach, the hierarchical clustering can be tracked with Lagrangian trajectories. It has been found that the intermittent scaling is related to the long time correlations in the particle acceleration, namely, the random forces

driving the particle motion is long range correlated (Mordant et al., 2002). It would be interesting to investigate the scaling in the Lagrangian scheme.

\section{Acknowledgments.}

T.-J.Z. is supported by the Fellowship of the World Laboratory. H.-Y.W., T.- 
J.Z. and L.-L.F. acknowledge support from the National Science Foundation of China (grants 10473002, 10573036 and 10545002). T.-J.Z thanks Xiang-Ping $\mathrm{Wu}$ for his valuable discussion. This work was also partially supported by US NSF AST 05-07340.

\section{References}

Adelberger, K. L., Steidel, C. C., Shapley, A. E., \& Pettini, M. 2003, ApJ, 584,45

Berera, A. \& Fang, L.-Z. 1994, Physical Review Letters, 72, 458

Bi, H., Fang, L.-Z., Feng, L., \& Jing, Y. 2003, ApJ, 598, 1

Bi, H. G., Boerner, G., \& Chu, Y. 1992, A\&A, 266, 1

Boldyrev, S., Linde, T., \& Polyakov, A. 2004, Physical Review Letters, 93, 184503

Boldyrev, S., Nordlund, Å., \& Padoan, P. 2002, Physical Review Letters, 89, 031102

Bonamente, M., Joy, M., LaRoque, S., Carlstrom, J., Nagai, D., \& Marrone, D. 2007, ArXiv e-prints, 708

Bonamente, M., Joy, M. K., LaRoque, S. J., Carlstrom, J. E., Reese, E. D., \& Dawson, K. S. 2006, ApJ, 647, 25

Cao, L., Chu, Y.-Q., \& Fang, L.-Z. 2006, MNRAS, 369, 645

Cole, S. \& Kaiser, N. 1988, MNRAS, 233, 637

Croston, J. H., Hardcastle, M. J., \& Birkinshaw, M. 2005, MNRAS, 357, 279

da Silva, A. C., Kay, S. T., Liddle, A. R., \& Thomas, P. A. 2004, MNRAS, 348, 1401

David, L. P., Slyz, A., Jones, C., Forman, W., Vrtilek, S. D., \& Arnaud, K. A. 1993, ApJ, 412, 479

Dolag, K., Hansen, F. K., Roncarelli, M., \& Moscardini, L. 2005, MNRAS, 363, 29

Dubrulle, B. 1994, Physical Review Letters, 73, 959

Edge, A. C. \& Stewart, G. C. 1991, MNRAS, 252, 428

Fang, L. Z. \& Thews, R. 1998, Wavelet in Physics (World Scientific, Singapore)

Feng, L.-L., Pando, J., \& Fang, L.-Z. 2003, ApJ, 587, 487

Feng, L.-L., Shu, C.-W., \& Zhang, M. 2004, ApJ, 612, 1

Haardt, F. \& Madau, P. 2001, in Clusters of Galaxies and the High Redshift Universe Observed in X-rays, ed. D. M. Neumann \& J. T. V. Tran

He, P., Feng, L.-L., \& Fang, L.-Z. 2004, ApJ, 612, 14

-. 2005, ApJ, 623, 601

He, P., Liu, J., Feng, L.-L., Shu, C.-W., \& Fang, L.-Z. 2006, Physical Review Letters, 96, 051302

Helsdon, S. F. \& Ponman, T. J. 2000, MNRAS, 315, 356

Jamkhedkar, P., Feng, L.-L., Zheng, W., \& Fang, L.-Z. 2005, ApJ, 633, 52

Jamkhedkar, P., Feng, L.-L., Zheng, W., Kirkman, D., Tytler, D., \& Fang, 
L.-Z. 2003, MNRAS, 343, 1110

Jamkhedkar, P., Zhan, H., \& Fang, L.-Z. 2000, ApJ, 543, L1

Jones, B. J. T. 1999, MNRAS, 307, 376

Kaiser, N. 1986, MNRAS, 222, 323

Kim, B., He, P., Pando, J., Feng, L.-L., \& Fang, L.-Z. 2005, ApJ, 625, 599

Kraichnan, R. H. 1968, Physics of Fluids, 11, 265

Lässig, M. 2000, Physical Review Letters, 84, 2618

Liu, J., Jamkhedkar, P., Zheng, W., Feng, L.-L., \& Fang, L.-Z. 2006, ApJ, 645,861

Liu, J.-R. \& Fang, L.-Z. 2008, ApJ, 672, 11

Lloyd-Davies, E. J. and Ponman, T. J. \& Cannon, D. B. 2000, MNRAS, 315, 689

Markevitch, M. 1998, ApJ, 504, 27

Matarrese, S. \& Mohayaee, R. 2002, MNRAS, 329, 37

Maughan, B. J. 2007, ApJ, 668, 772

McCarthy, I. G., Holder, G. P., Babul, A., \& Balogh, M. L. 2003, ApJ, 591, 526

Morandi, A., Ettori, S., \& Moscardini, L. 2007, MNRAS, 379, 518

Mordant, N., Delour, J., Léveque, E., Arnéodo, A., \& Pinton, J.-F. 2002, Physical Review Letters, 89, 254502

Nagai, D., Kravtsov, A. V., \& Vikhlinin, A. 2007, ApJ, 668, 1

Padoan, P., Boldyrev, S., Langer, W., \& Nordlund, Å. 2003, ApJ, 583, 308

Pando, J., Feng, L.-L., \& Fang, L.-Z. 2004, ApJS, 154, 475

Pando, J., Feng, L.-L., Jamkhedkar, P., Zheng, W., Kirkman, D., Tytler, D., \& Fang, L.-Z. 2002, ApJ, 574, 575

Peebles, P. J. E. 1980, The large-scale structure of the universe (Research supported by the National Science Foundation. Princeton, N.J., Princeton University Press, 1980. 435 p.)

Pen, U.-L. 1999, ApJ, 510, L1

Polyakov, A. M. 1995, Physical Review E, 52, 6183

Reese, E. D., Carlstrom, J. E., Joy, M., Mohr, J. J., Grego, L., \& Holzapfel, W. L. 2002, ApJ, 581, 53

Seljak, U. \& Zaldarriaga, M. 1996, ApJ, 469, 437

Shandarin, S. F. \& Zeldovich, Y. B. 1989, Reviews of Modern Physics, 61, 185

She, Z.-S. \& Leveque, E. 1994, Physical Review Letters, 72, 336

She, Z.-S. \& Waymire, E. C. 1995, Physical Review Letters, 74, 262

Sunyaev, R. A. \& Zeldovich, I. B. 1980, ARA\&A, 18, 537

Sutherland, R. S. \& Dopita, M. A. 1993, ApJS, 88, 253

Theuns, T., Leonard, A., Efstathiou, G., Pearce, F. R., \& Thomas, P. A. 1998, MNRAS, 301, 478

Tozzi, P. \& Norman, C. 2001, ApJ, 546, 63

Valageas, P. \& Silk, J. 1999, A\&A, 347, 1

Voit, G. M., Bryan, G. L., Balogh, M. L., \& Bower, R. G. 2002, ApJ, 576, 601

Wu, X.-P., Xue, Y.-J., \& Fang, L.-Z. 1999, ApJ, 524, 22

Xu, W., Fang, L.-Z., \& Wu, X.-P. 1998, ApJ, 508, 472 
Xue, Y.-J. \& Wu, X.-P. 2000, ApJ, 538, 65

-. 2003, ApJ, 584, 34

Zeldovich, Y. B. \& Sunyaev, R. A. 1969, Ap\&SS, 4, 301

Zhang, P. \& Pen, U.-L. 2003, ApJ, 588, 704

Zhang, T.-J., Liu, J., Feng, L.-l., He, P., \& Fang, L.-Z. 2006, ApJ, 642, 625 